\title{
Rechtliche Aspekte der Digitalisierung in der Medizin
}

\author{
Legal aspects of digitalization in the medical sector
}

\author{
A. Wienke, K. Friese
}

Die Digitalisierung unserer Gesellschaft, unseres Handelns und unseres Denkens wird allerorten diskutiert; in Anlehnung an ein literarisch bekannt gewordenes gallisches Dorf könnte man meinen, dass sich nur wenige Unbeugsame dem digitalen Zeitalter entgegenstemmen. Auch und gerade im Gesundheitswesen ist die $\mathrm{Di}$ gitalisierung derzeit eines der brennendsten Themen in der Gesundheitspolitik und in aktuellen Gesetzgebungsverfahren. So hat die Bundesregierung im Koalitionsvertrag vom 14.03.2018 festgehalten, dass bis 2020 ein Aktionsplan mit konkreten Maßnahmen entwickelt werden soll. Auch die aktuellen Diskussionen um die Einführung und den Nutzungsumfang der elektronischen Gesundheitskarte dominieren weiterhin in den Medien. Nicht zu vergessen ist auch die Debatte um die Lockerung des Fernbehandlungsverbotes, für welche sich eine große Mehrheit auf dem 121. Ärztetag im Mai 2018 ausgesprochen hat. Letztlich nimmt das Thema Digitalisierung durch die damit einhergehenden datenschutzrechtlichen Fragen an Fahrt auf; wer denkt dieser Tage dabei nicht an die EU-Datenschutzgrundverordnung (DSGVO) und die Neufassung des Bundesdatenschutzgesetzes (BDSG)?

Angesichts der Vielzahl von Themenkomplexen fällt es schwer, den Überblick zu behalten. Manch einer mag sich fragen, was nun genau unter dem Begriff „Digitalisierung“ zu verstehen ist. Eine allgemeinverbindliche Definition ist bisher nicht vorhanden.

\section{Was ist Digitalisierung?}

Der Begriff „Digitalisierung“ kann definiert werden als Oberbegriff für die aktuelle Entwicklung, Prozesse unter Einsatz moderner Technik und EDV zu überarbeiten und die Arbeitsabläufe, hier im Gesundheitswesen, dem aktuellen Stand der technischen Möglichkeiten anzupassen. Dazu gehören die elektronische Erfassung und Speicherung von Gesundheits- und Patientendaten, beispielsweise ein elektronischer Impf- oder Mutterpass oder die digitale Verwaltung des Zahn-Bonusheftes. Der Ausbau der elektronischen Gesundheitskarte, sicherer elektronischer Kommunikationswege und die Ermöglichung der Fernbehandlungen via Videochat sowie die Anwendung diverser Gesundheits-Apps unterfallen ebenfalls dem Begriff der Digitalisierung. Insgesamt sollen Abläufe vereinfacht und entbürokratisiert werden, wobei der Patient stets selbst Herr über seine Daten sein und bleiben soll.

Diese Anpassung kann nur gelingen, wenn sowohl die technischen als auch die rechtlichen Voraussetzungen vorhanden sind und der Wille der Nutzer, sich mit den neuen Abläufen vertraut zu machen. Einige Arztpraxen und Kliniken leisten auf diesem Gebiet bereits Pionierarbeit, andere lassen sich Zeit und warten ab, bis die drängendsten Fragen der Umsetzung beantwortet sind. Der Gesetzgeber hat in der Vergangenheit bereits die ersten Weichen für die Entwicklung der Digitalisierung gestellt, das sogenannte E-Health-Gesetz aus dem Jahr 2015 hat die Prozesse in maßgeblicher Weise angestoßen und beschleunigt.

\section{Das E-Health-Gesetz von 2015}

Das erste E-Health-Gesetz (Gesetz für sichere digitale Kommunikation und Anwendungen im Gesundheitswesen sowie zur Änderung weiterer Gesetze) trat am 29.12.2015 in Kraft. Der Gesetzgeber beabsichtigte mit diesem Regelwerk, die flächendeckende Einführung der Telematikinfrastruktur voranzutreiben. Im Vor- dergrund stand hier die elektronische Gesundheitskarte, die neben den stets aktuellen Versichertenstammdaten auch die Speicherung von Notfalldaten ermöglichen soll. Ein weiterer Schwerpunkt war, die digitale Infrastruktur von Arztpraxen zu verbessern. Arztbriefe sollen auf elektronischem Wege übermittelt und die elektronische Gesundheitsakte eingeführt werden. Der Heilberufeausweis kann mit einer elektronischen Signatur versehen werden, was die elektronische Kommunikation unter Sicherheitsaspekten erst ermöglicht. Insgesamt versprach sich der Gesetzgeber, durch die digitale Infrastruktur auch die Patientenrechte und die Patientensicherheit zu stärken. Der Patient soll dabei stets Herr seiner Daten sein und seine Mitwirkungsrechte - aber auch Mitwirkungspflichten - im Behandlungsverhältnis aktiv wahrnehmen.

Diese Entwicklung wird in Zukunft noch weiter vorangetrieben. Der Gesetzgeber beabsichtigt nun eine Weiterentwicklung der in 2015 gefassten Normierungen, es soll noch in der laufenden Legislaturperiode das „E-Health-Gesetz II“ kommen. Darin enthalten werden weitere Regelungen zur elektronischen Patientenakte, zur Fernbehandlung und zur Kostenverteilung sein.

\section{Die Umsetzung der Digitali- sierung in Klinik und Praxis}

Die angestrebte Digitalisierung in der Medizin erfordert eine ausreichende Telematikinfrastruktur. Mit der Umsetzung und Koordination ist die gematik - Gesellschaft für Telematikanwendungen der Gesundheitskarte mbH beauftragt worden. Hierbei handelt es sich um eine Gesellschaft mit beschränkter Haftung, die von den Spitzenorganisationen im Gesundheitswesen gegründet wurde (GKV-Spitzenverband, KBV, BÄK, BZÄK, DKG, KZBV u. a.). Im 
Zentrum der Entwicklungen stehen die Einrichtung der Telematikinfrastruktur und die elektronische Gesundheitskarte.

Die elektronische Gesundheitskarte ermöglicht zunächst das elektronische Stammdatenmanagement. Die allgemeinen Personendaten der Versicherten werden beim Einlesen der Karte online überprüft und nötigenfalls aktualisiert, beispielsweise bei einer Adressänderung. So kann sichergestellt werden, dass der behandelnde Arzt stets die aktuellen Stammdaten des Patienten hat. Später können auf der Karte auch medizinische Daten, z. B. Notfalldaten, gespeichert werden, die ausschließlich von Angehörigen der Heilberufe ausgelesen werden sollen. Auch Erklärungen wie Vorsorgevollmachten, Organspendeausweise und Patientenverfügungen können auf Wunsch des einzelnen Versicherten erfasst werden.

Seit dem 01.10.2016 haben gesetzlichversicherte Patienten einen Anspruch auf einen Medikationsplan, dieser ist den $\mathrm{Pa}$ tienten in Papierform auszuhändigen und regelmäßig zu aktualisieren ( $\$ 31$ a SGB V). Die Medikationspläne sollen aber auch auf der elektronischen Gesundheitskarte gespeichert werden. Dies soll die Patientensicherheit erhöhen, da wichtige Informationen damit auch nachbehandelnden Ärzten oder Notärzten zur Verfügung stehen.

Auch die Kommunikation zwischen den einzelnen Ärzten soll künftig digitalisiert ablaufen. Derzeit werden Arztbriefe analog verfasst und meist postalisch oder per Fax übermittelt. Künftig soll die Kommunikation ausschließlich auf elektronischem Wege erfolgen. Angesichts des Umstandes, dass es sich hier um besonders sensible Daten die der ärztlichen Schweigepflicht unterliegen - handelt, wurde die Fachanwendung KOM-LE (Sichere Kommunikation zwischen Leistungserbringern) entwickelt. Diese ist angebunden an das jeweilige Praxisverwaltungssystem bzw. an das jeweilige Krankenhaus-Informationssystem. Die Daten werden unmittelbar zwischen den teilnehmenden Praxen bzw. Kliniken verschlüsselt übersandt. Neben der Zeitersparnis auf dem Übertragungsweg soll so auch der Datenschutz gewährleistet werden.
Die Lockerung des Fernbehandlungsverbotes wird die Kommunikation zwischen Ärzten und Patienten künftig weitgehend verändern. Seit dem 1. April 2017 sind bereits Videosprechstunden grundsätzlich möglich (§291 a SGB V i. V. m. Anlage 31 b zum Bundesmantelvertrag-Ärzte). Nach der Mehrheitsentscheidung auf dem 121. Deutschen Ärztetag, in der sich auch die Ärzteschaft für eine Lockerung des Fernbehandlungsverbotes ausgesprochen hat, hat die Landesärztekammer Sachsen bereits reagiert. Die Berufsordnung der Ärzte in Sachsen wird voraussichtlich zum 01.09.2018 geändert. Künftig wird es nach der Berufsordnung Sachsen erlaubt sein, im Einzelfall eine Behandlung oder Beratung über digitale Kommunikationsmedien durchzuführen. Freilich muss auch hierbei - besonders aus haftungsrechtlichen Gründen - die ärztliche Sorgfalt und Fürsorge stets gewahrt bleiben.

Die Einführung einer elektronischen Gesundheitsakte ist ebenfalls ein wichtiger Pfeiler bei der Digitalisierung. In dieser Datenbank sollen die Gesundheitsdaten der Patienten, wie Allergien, Anamnesen, Krankengeschichte, Medikamente, Verordnungen und Überweisungen sowie weitere Informationen bundesweit zentral gespeichert werden. Der Zugang soll - im Einverständnis des Patienten - mit der elektronischen Gesundheitskarte in Verbindung mit dem jeweiligen Heilberufeausweis des Arztes möglich sein. Die Karten fungieren insoweit als Zugangsschlüssel. So wird sichergestellt, dass nur befugte Personen Zugang zu den Daten haben. Dabei ist es nach dem Willen des Gesetzgebers letztlich immer der Patient, der die Hoheit über die Daten ausübt und entscheidet, ob und welche Daten in der Akte gespeichert oder auch gelöscht werden. Die bekannte - und von § 630 f BGB gesetzlich vorgeschriebene - Behandlungsdokumentation wird hierdurch nicht ersetzt. Vielmehr bündelt die elektronische Gesundheitsakte die Informationen verschiedener Praxen und gewährt so bestenfalls einen Überblick über den Gesundheitsstatus des Patienten.

Neben diesen Schwerpunkten der neuen Regelungen nutzen viele Arztpraxen und Kliniken eine Vielfalt digitaler Anwendungen bereits selbstverständlich in der täglichen Praxis: So wird vielerorts die
Patientendokumentation unmittelbar elektronisch festgehalten anstatt sie handschriftlich aufzuschreiben. Einige Praxen und Kliniken digitalisieren ihre analogen Daten nachträglich zu Aufbewahrungszwecken. Aufklärungsgespräche werden mit Unterstützung von digitalen Bögen geführt und Unterschriften zur Einwilligung auf dem Tablet geleistet.

Auch aus juristischer Sicht birgt die Digitalisierung im Gesundheitswesen neue Chancen, eröffnet aber auch ganz neue Fragestellungen und Haftungsrisiken.

\section{Chancen und Risiken der Digitalisierung}

Nach dem Willen des Gesetzgebers soll der Patient die vollständige Verfügungsgewalt über seine personenbezogenen (Gesundheits-) Daten haben. Dieser Ansatz ist Ausfluss des Selbstbestimmungsrechts der Patienten, welches in der Medizin immer weiter in den Fokus rückt. Es ist mittlerweile Standard, dass sich viele Patienten vor oder nach dem Arztbesuch über die Erkrankung und Therapiemaßnahmen selbst informieren und gezielte Fragen an ihren Arzt stellen. Dies verändert die Gesprächsführungen und die Anforderungen an die Aufklärung enorm. Die konkrete Patienteninformation durch den Arzt spielt - auch vor dem Hintergrund des Arzthaftungsrechts inzwischen eine entscheidende Rolle im Arzt-Patienten-Verhältnis. Der Arzt ist gehalten, den Patienten bei der Ausübung seines Selbstbestimmungsrechts zu unterstützen und ihm zu helfen, die medizinischen Informationen zu verarbeiten und zu bewerten. Die Patienten erhalten aber nicht nur mehr Rechte, sondern sie sind zukünftig auch dazu angehalten, sich mehr mit Gesundheitsbelangen zu befassen und eigenverantwortlich zu handeln. Nur bei entsprechender Patienten-Compliance können beide Seiten - Arzt und Patient - von den neuen Entwicklungen profitieren.

Ein besonders brisanter Aspekt bei der Entwicklung der Digitalisierung ist der Datenschutz (data privacy). Mit der am 25. Mai 2018 in Kraft getretenen EU-Datenschutzgrundverordnung (DSGVO) wurden die bisher geltenden Datenschutzregelungen umfassend novelliert. Durch die den Arzt- 
praxen und Kliniken darin auferlegten Verpflichtungen wurde der Datenschutz auch wieder verstärkt ins Bewusstsein gerufen. Ärzte sind generell - auch vor dem Hintergrund der ärztlichen Schweigepflicht, vgl. §203 Strafgesetzbuch, - verpflichtet, mit den Daten ihrer Patienten sensibel umzugehen. Die neuen Regelungen sollten daher zum Anlass genommen werden, Prozesse und Abläufe zu prüfen und gegebenenfalls anzupassen. Gerade hier bietet die Nutzung digitaler Anwendungen aber auch große Chancen, wie beispielsweise die elektronische Kommunikation via KOM-LE. Datenschutzrechtliche Fragestellungen wird die neue Telematikinfrastruktur jedenfalls in großer Zahl aufwerfen. Dies beginnt bereits bei der Frage, wer auf die auf der elektronischen Gesundheitskarte gespeicherten medizinischen Daten überhaupt zugreifen darf und wie lange diese dort gespeichert bleiben sollen. Gleiches gilt für die elektronische Patientenakte. Die Einhaltung des Datenschutzes ist unumgänglich und sollte - angesichts der drohenden Bußgelder von jedem Arzt ernst genommen werden. Bei der Einführung von neuen digitalen Anwendungen in der Arztpraxis sollte daher immer auch ein datenschutzrechtlicher Check-up erfolgen; zudem sind die Mitarbeiter im Umgang mit den sensiblen personenbezogenen Daten der Patienten zu schulen und zu sensibilisieren. Der unbefugte Zugriff auf Daten muss durch entsprechende technische Maßnahmen (TOM 's) verhindert werden, dies kann einen nicht zu unterschätzenden Aufwand bedeuten.

Die Digitalisierung wird auch im Arzthaftungsrecht große Bedeutung erlangen. Ärzte müssen bei dem Umgang mit digitalen Anwendungen auch immer berücksichtigen, welche Haftungsrisiken aus ihnen erwachsen. Die einzelne Arztpraxis wird die internen Abläufe entsprechend anpassen müssen. Über die elektronische Kommunikation eingehende Arztbriefe und Informationen müssen von dem Arzt auch tatsächlich (zeitnah) zur Kenntnis genommen werden. Medikationspläne sind stets zu aktualisieren ( $\$ 31$ a Abs. 3 SGB V), ebenso wie die Notfalldaten. Dies wirft auch die Frage nach der Zuständigkeit für die Aktualisierung der Daten auf. Ist jeder Arzt verpflichtet, den Patienten auf etwaige neue Umstände zu befragen bzw. zu untersuchen oder darf er sich uneingeschränkt auf die vormals gespeicherten Daten verlassen? Und in welchem Umfang muss der Arzt diese überhaupt zur Kenntnis nehmen, muss der Arzt die Behandlungsunterlagen des Psychotherapeuten vollständig zur Kenntnis nehmen, wenn er die elektronische Gesundheitsakte liest? Wer haftet, wenn die Daten nicht aktualisiert worden sind - der ursprünglich dokumentierende Arzt oder vielleicht der nachbehandelnde Arzt, der aber ein ganz anderes Fachgebiet hat und dem sich der Patient nur wegen einer ganz spezifischen Erkrankung vorstellt? Oder hat der Patient selbst ein Mitverschulden, wenn er wichtige Daten aus seiner elektronischen Gesundheitsakte löscht bzw. nicht aufnehmen lässt? Im Falle eines Notfalls muss überhaupt erst einmal sichergestellt werden, dass der jeweils behandelnde Arzt die auf der elektronischen Gesundheitskarte gespeicherten Daten auch zur Kenntnis nimmt und diese bei der Behandlung berücksichtigt. Die Digitalisierung wird daher auch das Arzthaftungsrecht nicht unberührt lassen und neue Fragestellungen aufwerfen, welche die Jurisprudenz der kommenden Jahre entscheiden und weiterentwickeln wird. Wahrscheinlich werden zukünftig die Sorgfaltsverpflichtungen der Ärzte eine neue Gestalt erhalten, auch im Hinblick auf nun mögliche Fernbehandlungen. Denkbar ist auch, dass Patienten auch unter haftungsrechtlichen Gesichtspunkten selbst eine höhere Mitverantwortlichkeit erfahren werden.

Natürlich kommt es in der ärztlichen Praxis vor, dass Streitigkeiten aus einem Behandlungsverhältnis vor Gericht ausgetragen werden müssen. Dies kann beispielsweise der klassische Arzthaftpflichtfall sein, in dem der Patient Ansprüche wegen eines Behandlungsfehlers geltend macht, oder aber die Klage des Arztes gegen den Patienten auf Zahlung seines Honorars. Das geltende Prozessrecht ist für den Ausgang eines Verfahrens von großer Bedeutung. Die prozessualen Beweislastregelungen und die Beweisbarkeit eines Tatsachenvortrages sind streitentscheidend. Als bedeutendste Beweismittel in einem solchen Zivilprozess dienen natürlich der Sachverständige und die Zeugen, aber auch Urkunden - wie die Behandlungsdokumentation oder eine Gebührenvereinbarung - spielen eine wesentliche Rolle. Die geltenden Formvorschriften sind derzeit bei weitem nicht an die technischen Möglichkeiten angepasst. Besteht eine gesetzliche Schriftformerfordernis, bspw. nach $\S 2$ GOÄ oder bei IGeL-Leistungen ( $§ 18$ Abs. 8 BMV-Ä), ist besondere Vorsicht geboten. Die gesetzliche Schriftform erfordert derzeit noch die handschriftliche Unterschrift auf einem körperlichen Dokument, oder aber eine elektronische Signatur, die kaum ein Patient haben wird. Digitale Unterschriften, bspw. auf einem Tablet, erfüllen diese Voraussetzungen nicht. Werden Schriftstücke nachträglich digitalisiert und die Originale vernichtet, so besteht zudem ein erhebliches Risiko, dass der Beweis im Fall der Fälle nicht gelingt. Denn die Vorlage des Originals ist für das Führen eines Urkundenbeweises unerlässlich. Digitalisierte Schriftstücke wird ein Gericht im Regelfall zwar nicht unberücksichtigt lassen, jedoch ist es ohne die Vorlage des Originals für die Gegenseite wesentlich einfacher, die Beweiskraft zu erschüttern. Vor der Vernichtung der Originalunterlagen sollte daher bestenfalls ausgeschlossen sein, dass diese noch Gegenstand eines Streitfalls werden können. Ähnlich wie die Medizin steht auch die Jurisprudenz vor einem digitalen Wandel. Die elektronische Akte und elektronische Kommunikation mit Gerichten und Behörden wird auch dort bald zum Standard gehören.

\section{Ausblick}

Die Digitalisierung im Gesundheitswesen schreitet voran und wird Arbeitsabläufe und Prozesse in den Arztpraxen und Kliniken umfangreich verändern. Die Anwendungen haben dabei das Potential, Abläufe zu vereinfachen und damit letztlich Zeit zu sparen. Eine besondere Bedeutung kommt dem Selbstbestimmungsrecht der Patienten zu. Jede Nutzung digitaler Anwendungen wird sich auch immer an dem Patientenwillen und den datenschutzrechtlichen Regelungen messen lassen müssen. Bisher allgemein geltende Regelungen, insbesondere solche des Prozessrechts, werden an die neuen technischen Möglichkeiten angepasst werden müssen. Eine Weiterentwicklung wird auch die Rechtsprechung selbst erfahren, insbesondere in Fragen des Arzthaftungsrechts werden noch nie dagewesene Sachverhalte richterlich entschieden werden müssen. Ob sich mit der zunehmend Platz greifenden Digitalisierung im 
Gesundheitswesen auch Verbesserungen in der Patientenversorgung ergeben werden, wird zwar von den Fürsprechern behauptet, muss sich in der Praxis aber erst noch beweisen.

\section{Zusammenfassung}

Die Digitalisierung im Gesundheitswesen umschreibt die Anpassung der täglichen Abläufe und Prozesse an die aktuellen technischen Möglichkeiten und Kommunikationswege. Aspekte der Datensicherheit und das Selbstbestimmungsrecht der Patienten stehen dabei im Fokus. Der Gesetzgeber selbst treibt die Entwicklung der Digitalisierung voran, neben den bestehenden Regelungen werden in der laufenden Legislaturperiode weitere Neuerungen erfolgen. Die Digitalisierung wird die Abläufe im ärztlichen Alltag verändern. Dabei werden auch ganz neue, rechtliche Fragestellungen in den Fokus rücken. Reformen des materiellen Rechts und des Prozessrechts werden erforderlich sein. Die Jurisprudenz wird die neuen Fragestellungen letztlich lösen, was aber auch zu einer Fortentwicklung des an die ärztliche Sorgfaltspflicht zu stellenden Maßstabs führen wird. Letztlich wird sich kein Arzt der Entwicklung verschließen können.

\section{Summary}

The buzz phrase "digitalisation in the health sector" describes the adaptation of daily routines and processes to current technical possibilities and means of communication. Data protection and self-determinati- on of patients are at the focal point in this process. The legislator keeps propelling the development of digitalisation: Apart from already existing regulations, further legal novelties are expected to come into being in the subsequent legislative period. Digitalisation is bound to alter the day-to-day processes of medical practitioners. In that context completely new legal question are likely to emerge. Reforms of substantive law as well as of procedural law will become necessary. The jurisdiction will have to solve the arising legal problems; this might eventually also lead to an alteration of the criteria of medical due diligence. In the end, these developments will affect every medical practitioner.

\section{Erstveröffentlichung}

Erstveröffentlicht in der „OUP - Orthopädische und Unfallchirurgische Praxis“ 9/2018

\section{Korrespondenzadresse}

Wienke \& Becker - Köln
Rechtsanwälte
Sachsenring 6
50677 Köln
awienke@kanzlei-wbk.de

Bibliografie

DOI https://doi.org/10.1055/a-0652-6505 Laryngo-Rhino-Otol 2018; 97: 713-716 (c) Georg Thieme Verlag KG Stuttgart · New York ISSN 0935-8943 